PDFlib PLOP: PDF Linearization, Optimization, Protection

Page inserted by evaluation version www.pdflib.com - sales@pdflib.com 


\title{
The Amygdala, Synaptic Plasticity, and Fear Memory
}

\author{
STEPHEN MAREN \\ Department of Psychology and Neuroscience Program, University of Michigan, \\ Ann Arbor, Michigan 48109-1109, USA
}

\begin{abstract}
The nature and mechanisms of synaptic plasticity in the amygdala and the relation of amygdaloid plasticity to behavior are exciting new areas of study in neuroscience. These issues were at the heart of presentations by Paul Chapman, Michael Fanselow, Patricia Shinnick-Gallagher, and Michael Rogawski in a session entitled "Long-Term Plasticity in Amygdala Synaptic Transmission" that was held at the conference featured in this volume. In this chapter, I briefly summarize these talks and give my perspective on the presentations as the session chair. I argue that we must first understand the role of the amygdala in learning and memory in order to understand the contribution of amygdaloid synaptic plasticity to behavior. Although it is generally agreed that the amygdala is involved in several forms of emotional learning and memory such as pavlovian fear conditioning, a recent debate has emerged concerning the precise role of the amygdala in learning versus performing fear responses. I discuss data from my laboratory that unravel this issue. I argue that the basolateral complex of the amygdala (BLA) normally plays an essential role in associative processes in fear conditioning. Nonetheless, rats with BLA lesions acquire and express conditional fear under some conditions. A neuroanatomical model that accounts for these data is presented.
\end{abstract}

KEYWORDs: overtraining; lesion; basolateral nucleus; NMDA; freezing; fear conditioning; rat

\section{INTRODUCTION}

What is the role of synaptic plasticity in the amygdala in behavior? This is the question a group of prominent neuroscientists addressed in a session entitled "LongTerm Plasticity in Amygdala Synaptic Transmission" presented at the New York Academy of Sciences conference that is reported in this volume. This session featured illuminating presentations by Paul Chapman from Cardiff University, Michael Fanselow from the University of California at Los Angeles, Patricia ShinnickGallagher (a conference organizer) from the University of Texas Medical Branch, and Michael Rogawski from the National Institutes of Health; I served as the chair of the session. Because each of the presentations is recapitulated in subsequent chap-

Address for correspondence: Stephen Maren, Department of Psychology, University of Michigan, 525 E. University Ave., Ann Arbor, MI 48109-1109. Voice: 734-936-6532; fax: 734-7637480 .

maren@umich.edu

Ann. N.Y. Acad. Sci. 985: 106-113 (2003). ( $) 2003$ New York Academy of Sciences. 
ters in this volume, I do not consider them in depth here. Rather, I focus on some of the issues that cut across all of the presentations, and I summarize some of my own work that is relevant to these issues. I will also distill some of the major points that were considered in the discussion that followed the formal presentations.

An important theme that guided the talks in our session as well as many of the presentations featured in the 3-day conference is the capacity of amygdala neurons for enduring plasticity. For example, Chapman focused on tetanus-induced longterm potentiation (LTP) in the basolateral nucleus of the amygdala and compared and contrasted amygdaloid and hippocampal LTP in normal and genetically modified mice. Fanselow argued that synaptic plasticity in the basolateral complex of the amygdala (BLA) is responsible for storing long-term memories of pavlovian fear conditioning. Shinnick-Gallagher revealed that fear learning produces changes in synaptic transmission in the BLA in vitro that share properties with tetanus-induced LTP. Rogawski introduced a novel and slowly developing form of synaptic plasticity in the BLA that is induced by activation of the kainate-sensitive class of glutamate receptors. Although there was consensus among the presenters that amygdala neurons exhibit long-term synaptic plasticity and that this plasticity has a role in behavior, discussion revealed that the relation between various forms of amygdaloid plasticity and behavior is unclear (see below).

The presenters in this session represented a diversity of approaches to the study of local synaptic plasticity within the amygdala and its relation to behavior. Chapman used genetically modified mice to explore correlations between synaptic physiology in vitro and behavior in several memory tasks. Shinnick-Gallagher moved in the opposite direction, examining the consequences of fear learning for synaptic transmission in amygdala brain slices. Fanselow used permanent or temporary brain lesions in intact animals to examine the role of the amygdala in fear learning and memory. Rogawski used pharmacological techniques in brain slices to assess the role amygdaloid glutamate receptors play in synaptic plasticity and epileptogenesis. Ultimately, all of these approaches are necessary to provide an integrated picture of amygdaloid physiology and behavior.

An important problem that frames the debate about amygdaloid synaptic plasticity and behavior concerns the role of the amygdaloid nuclei in learning and memory. Indeed, as many chapters in this volume attest, there is broad consensus that the amygdala has an important role in fear and in forms of learning and memory that are motivated by fear. Indeed, Fanselow argued in his presentation that the amygdala is a storehouse for fear memories. One piece of evidence that he used to support this claim was his finding that BLA lesions made over a year after pavlovian fear conditioning produced a retention deficit for the old fear memory that was comparable to the deficit for a more recent fear memory. Importantly, Fanselow demonstrated that rats with BLA lesions could exhibit fear behavior (in this case, freezing) using an overtraining procedure developed in my laboratory. ${ }^{1}$ Playing the devil's advocate, Mike Davis from Emory University asked if the freezing deficit in rats with BLA lesions was truly a memory deficit or a deficit in performing the fear response. Davis suggested that an overtraining manipulation might not clarify matters because of compensation of brain structures not normally involved in mediating fear learning.

Obviously, it is critical to differentiate the role of the BLA in learning versus performing fear responses, such as freezing, in order to understand the role of amygdaloid synaptic plasticity in behavior. My laboratory has directly addressed this issue, 
and I described these data to lead off the group discussion in our session. I will summarize these data in the remainder of the chapter. We have used a pavlovian fearconditioning paradigm to examine the effect of neurotoxic BLA lesions on the acquisition and expression of conditional freezing behavior. In this paradigm, rats are presented with conditioning trials in which a neutral stimulus (a conditional stimulus, CS) is paired with an aversive unconditional stimulus (US). After conditioning, the CS comes to elicit a variety of learned fear responses (conditional responses, CRs). In addition, the conditioning context (the place where conditioning occurs) will elicit fear CRs.

Considerable evidence indicates that the BLA is essential for both the acquisition and expression of a variety of fear responses, including freezing, ${ }^{2-5}$ tachycardia and hypertension, ${ }^{6}$ hypoalgesia, ${ }^{7}$ and fear-potentiated startle. ${ }^{8-10}$ Deficits in conditional freezing are obtained even when BLA lesions are made more than a month ${ }^{2,10}$ or a year (Fanselow, unpublished data presented in session) after fear conditioning.

An important issue is whether BLA lesions affect the expression of unlearned or unconditional fear responses (URs). Data are mixed. In some cases, BLA manipulations affect the expression of fear URs. For example, inactivation of BLA neurons with a glutamate receptor antagonist attenuates the increase in the acoustic startle reflex produced by bright light. ${ }^{11}$ In other cases, BLA lesions produce deficits in freezing CRs without affecting the freezing UR elicited, for example, by a predator odor.

Involvement of the BLA in the expression of URs has led some to question the hypothesis that the BLA is uniquely involved in fear learning and memory. ${ }^{12}$ Particular concern has been voiced over the measurement of freezing behavior as an index of fear, because some reports indicate that amygdala damage can increase locomotor activity and reduce freezing behavior independently of conditioning. Indeed, it has been argued that the freezing deficits obtained in rats with BLA lesions may be due solely to deficits in performing the freezing response, not in establishing the fear memories. ${ }^{13}$

Although freezing behavior is exhibited by rats with neurotoxic BLA lesions under some conditions, ${ }^{5}$ it is not clear if animals with BLA lesions have the capacity to exhibit freezing following footshock. To address this issue, we performed a series of experiments in which we delivered much more training than is typically used to establish freezing CRs, a so-called overtraining procedure. ${ }^{1,3}$ We used a contextual fear-conditioning procedure in which footshock was delivered in a novel context, that is, the shock was not signaled by a discrete CS. A critical variable that we manipulated was the timing of BLA lesions with respect to training. Rats were given neurotoxic BLA lesions either 1 week before overtraining to assess the effect of the lesions on the acquisition of conditional freezing or 1 day after overtraining to assess the effect of the lesion on the expression of conditional freezing. We suspected that rats with pretraining lesions might recruit an alternate neural system to acquire conditional fear and that this would be unmasked by overtraining. By contrast, we hypothesized that the BLA encodes and stores fear memories in intact rats, even after overtraining. Thus, we expected that posttraining BLA lesions would devastate conditional freezing independent of the level of training. This hypothesis assumes that redundant associative centers are present in the brain, but that the BLA has primacy in the intact animal.

The results confirmed our predictions. As shown in FIgURE 1A, rats with pretraining lesions exhibited robust deficits in conditional freezing after minimal train- 

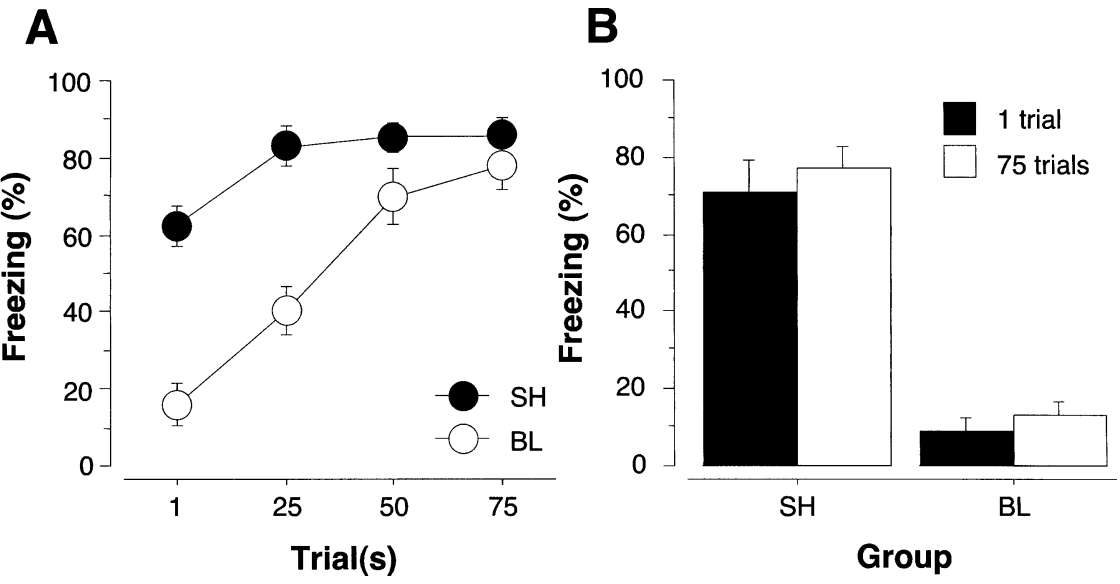

FIGURE 1. (A) Pretraining basolateral amygdala (BLA) lesions and overtraining. Mean $( \pm$ SEM) percentage of freezing for sham-operated rats $(\mathrm{SH})$ or rats with neurotoxic basolateral amygdala lesions (BL) after contextual fear conditioning consisting of $1,25,50$, or 75 trials. Rats with BL lesions acquire contextual freezing after extensive overtraining. (B) Posttraining BLA lesions and overtraining. Mean ( \pm SEM) percentage of freezing for sham-operated rats $(\mathrm{SH})$ or rats with neurotoxic basolateral amygdala lesions (BL). Surgery was performed 24 hours after contextual fear conditioning consisting of 1 or 75 trials. Extensive overtraining before surgery does not mitigate the effects of BLA lesions. Adapted with permission from Ref. 1 (copyright 1999 by the Society for Neuroscience).

ing ( 1 or 25 trials), but they acquired conditional freezing after overtraining (50 or 75 trials; Experiment 1). ${ }^{1}$ Importantly, these data indicate that when given enough training, rats with BLA lesions are capable of exhibiting shock-elicited freezing. The rats are capable of both learning (albeit slowly) and performing the conditional freezing response. By contrast, rats with posttraining BLA lesions exhibited low levels of freezing despite extensive overtraining prior to surgery (FIG. 1B; Experiment 2). ${ }^{1}$ Because rats with BLA lesions are capable of freezing, it is unlikely that the deficits observed in rats with posttraining lesions are due to a performance deficit. Alternatively, the results suggest that posttraining BLA lesions disrupted the memory for fear conditioning.

If posttraining BLA lesions impair the memory for fear conditioning, then rats with BLA lesions should not exhibit "savings" (i.e., spared memory). To test this possibility, we examined whether the reacquisition of conditional freezing was accelerated in BLA rats that had been overtrained. Such an accelerated rate of reacquisition would provide evidence for savings of fear memory. However, as shown in FIgURE 2, we found that rats with BLA lesions reacquired conditional freezing at the same rate, regardless of the amount of presurgical training (Experiment 2). ${ }^{1}$ Thus, we found no evidence for savings of fear memory in rats with posttraining BLA lesions. Importantly, the deficits in conditional freezing that we have observed are not due to changes in shock reactivity or to elevations in locomotor activity. ${ }^{2,3}$

In many pavlovian fear-conditioning paradigms, discrete CSs such as tones or lights are paired with the aversive US. Such CSs elicit conditional fear responses, 


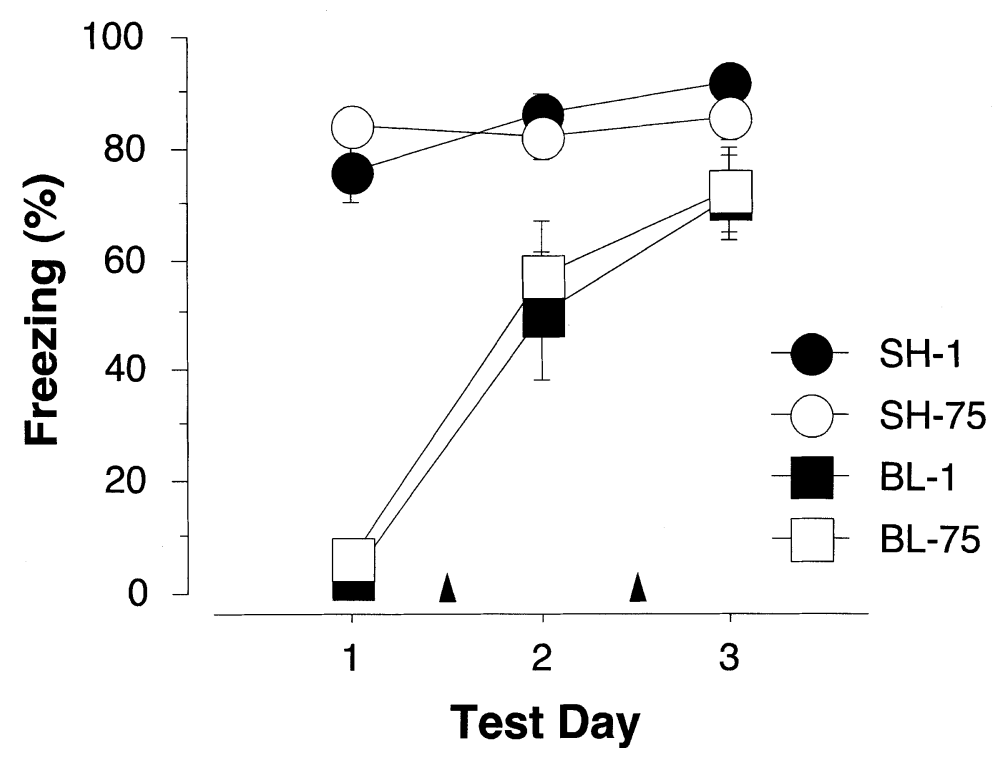

FIGURE 2. Reacquisition of contextual freezing in rats with posttraining basolateral amygdala (BLA) lesions. Mean ( \pm SEM) percentage of freezing for sham-operated rats $(\mathrm{SH})$ or rats with neurotoxic basolateral amygdala lesions (BL) during 3 posttraining periods. Period 1 re-plots the data in FIGURE 1B. Twenty-five context conditioning trials were interposed between periods 1 and 2 and periods 2 and 3 (arrowheads), for a total of 50 additional trials. Note the similar rates of reacquisition in BLA rats originally trained with 1 or 75 conditioning trials; there is no evidence for savings in overtrained BLA rats. Adapted with permission from Ref. 1 (copyright 1999 by the Society for Neuroscience).

including freezing, in a manner that is similar to contexts associated with footshock. Because rats with pretraining BLA lesions acquire conditional freezing following extensive overtraining, we were interested in examining whether they would also acquire fear to a discrete CS. Rats were administered 75 tone-footshock conditioning trials either 1 week after receiving neurotoxic BLA lesions or 1 day before the same lesions. Surprisingly, BLA lesions abolished conditional freezing to the tone CS independent of whether they were made before or after overtraining. Yet, rats with pretraining lesions still acquired contextual fear. Hence, rats with pretraining BLA lesions exhibited fear to the place where conditioning occurred, but completely lacked fear of the auditory CS that predicted shock onset. ${ }^{1}$

Considering all of these results, it is reasonable to conclude that BLA damage is not essential for the performance of freezing behavior. Rather, the BLA appears to have a critical role in associative processes underlying pavlovian fear conditioning. Indeed, BLA lesions produce a near global deficit in learning and memory that can only be overcome under a narrow set of conditions. Specifically, rats with BLA lesions slowly condition fear to contexts after overtraining, although it requires more than 10 times the amount of training required to produce maximal fear in normal animals. Nevertheless, they are unable to acquire fear to acoustic stimuli or retain 


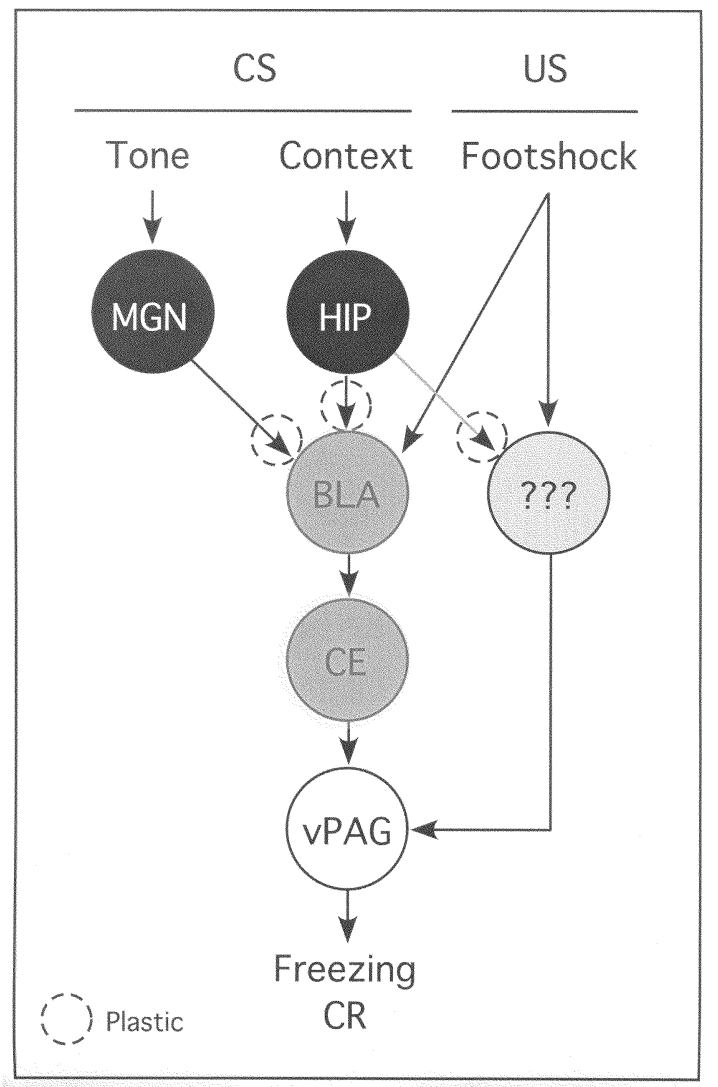

FIGURE 3. Hypothetical circuit model illustrating pathways conveying information concerning acoustic and contextual conditional stimuli (CSs), the footshock unconditional stimulus (US), and the freezing conditional response (CR). The CS and US converge in the basolateral complex of the amygdala (BLA), and the CS input to the BLA is robust and plastic. Convergence of the CS and US is also posited to occur in an unknown extra-amygdaloid structure (???), and it is proposed that weak CS inputs to this structure originate from the hippocampus. There are no auditory CS inputs to this structure. Plastic inputs that undergo long-term potentiation during fear conditioning are indicated by the dashed circles. Abbreviations: CE, central nucleus of the amygdala; HIP, hippocampal formation; MGN, medial geniculate nucleus of the thalamus; vPAG, ventral division of the periaqueductal gray; ???, unknown point of contextual CS and US convergence.

overtrained fear memories to contexts or tones if the lesions occur after the acquisition of such memories. Even when rats with BLA lesions acquire contextual fear, it is unclear if these memories are long lasting. Further work will be directed at this issue.

What accounts for context fear acquisition in rats with pretraining BLA lesions? One possibility is that the small numbers of BLA neurons that survive the lesion mediate context fear conditioning. These neurons may be more likely to receive dis- 
tributed multimodal contextual information than more localized acoustic information. However, rats with much larger lesions of the amygdala that include the BLA and adjacent amygdaloid nuclei also acquire contextual freezing. ${ }^{3}$ Another possibility is that neurons outside the amygdala mediate fear conditioning in the absence of the BLA. Because these extra-amygdaloid neurons do not acquire fear in the intact animal, we propose that they only receive a weak contextual CS input. Hence, in the intact animal, the BLA is more effective than these neurons in acquiring associative strength because it has robust CS inputs. In the absence of the BLA, these alternate neurons acquire contextual fear at the slow rate afforded by a weak input. A hypothetical model illustrating these points is shown in FIGURE 3.

The critical involvement of the BLA in the acquisition and expression of fear memories is important for understanding the contribution of synaptic plasticity in the BLA to behavior. As just described, there is now strong reason to believe that amygdaloid synaptic plasticity mechanisms, such as LTP, are involved in encoding new fear memories. The session presenters have elaborated some of the cellular mechanisms of various amygdaloid plasticity mechanisms that may contribute to behavior in this way. Collectively, this work serves as an important foundation for further studies aimed at elaborating the role of amygdaloid synaptic plasticity in learning and memory.

\section{ACKNOWLEDGMENTS}

This work was supported by National Institute of Mental Health Grant R29MH57865.

\section{REFERENCES}

1. MAREN, S. 1999. Neurotoxic lesions of the basolateral amygdala impair learning and memory but not the performance of conditional fear in rats. J. Neurosci. 19: 86968703.

2. Maren, S. et al. 1996. Retrograde abolition of conditional fear after excitotoxic lesions in the basolateral amygdala of rats: absence of a temporal gradient. Behav. Neurosci. 110: 718-726.

3. Maren, S. 1998. Overtraining does not mitigate contextual fear conditioning deficits produced by neurotoxic lesions of the basolateral amygdala. J. Neurosci. 18: 30883097.

4. Cousens, G. \& T. Отто. 1998. Both pre- and posttraining excitotoxic lesions of the basolateral amygdala abolish the expression of olfactory and contextual fear conditioning. Behav. Neurosci. 112: 1092-1103.

5. Wallace, K.J. \& J.B. Rosen. 2001. Neurotoxic lesions of the lateral nucleus of the amygdala decrease conditioned fear but not unconditioned fear of a predator odor: comparison with electrolytic lesions. J. Neurosci. 21: 3619-3627.

6. LeDoux, J.E. et al. 1990. The lateral amygdaloid nucleus: sensory interface of the amygdala in fear conditioning. J. Neurosci. 10: 1062-1069.

7. Helmstetter, F.J. 1992. The amygdala is essential for the expression of conditional hypoalgesia. Behav. Neurosci. 106: 518-258.

8. SANANES, C.B. \& M. DAvis. 1992. $N$-methyl-D-aspartate lesions of the lateral and basolateral nuclei of the amygdala block fear-potentiated startle and shock sensitization of startle. Behav. Neurosci. 106: 72-80. 
9. CAMPEAU, S. \& M. DAvis. 1995. Involvement of the central nucleus and basolateral complex of the amygdala in fear conditioning measured with fear-potentiated startle in rats trained concurrently with auditory and visual conditioned stimuli. J Neurosci. 15: $2301-2311$

10. Lee, Y. et al. 1996. Lack of a temporal gradient of retrograde amnesia following NMDA-induced lesions of the basolateral amygdala assessed with the fear-potentiated startle paradigm. Behav. Neurosci. 110: 836-839.

11. Walker, D.L. \& M. Davis. 1997. Double dissociation between the involvement of the bed nucleus of the stria terminalis and the central nucleus of the amygdala in startle increases produced by conditioned versus unconditioned fear. J. Neurosci. 17: 93759383.

12. CAHILL, L. et al. 1999. Is the amygdala a locus of "conditioned fear"? Some questions and caveats. Neuron 23: 227-228.

13. Vazdarjanova, A. \& J.L. MCGaUGH. 1998. Basolateral amygdala is not critical for cognitive memory of contextual fear conditioning. Proc. Natl. Acad. Sci. USA 95: $15003-15007$. 